\title{
Non-singleton Fuzzy Sets for Disturbance Attenuation
}

\author{
Andras Simon and George T. Flowers ${ }^{\dagger}$ \\ Department of Mechanical Engineering, Auburn University, Auburn, Alabama 36849, USA
}

(Received 3 March 2006; revised 11 June 2007; accepted 10 September 2007)

\begin{abstract}
Active magnetic bearings provide non-contact levitation of a ferromagnetic object with controllable damping and spring constant. These nonlinear systems require active control, as the electromagnetic levitation is unstable by nature. Linear control methods have limited success in the face of such a typically nonlinear system and the presence of modelling inaccuracies often force one to consider other control strategies. Fuzzy logic control performs very well in nonlinear control situations where the plant parameters are either partially or mostly unidentified. Its effectiveness for nonlinear systems also offers advantages to magnetic bearing systems. Uncertainties originate from several sources in the fuzzy logic controller, ranging from rule definitions to shape and location of the membership functions as well as unreliable input measurements. Non-singleton fuzzy set inputs allow one to account for input measurement uncertainty due to noise and other disturbances. The current work consists of model development, controller design, simulation and experimental validation. The controller designs are implemented and tested using a bench-top rotor rig equipped with a rotor supported by a radial magnetic bearing. Some representative results are presented and discussed.
\end{abstract}

${ }^{\dagger}$ Member of the International Institute of Acoustics and Vibration (IIAV)

\section{INTRODUCTION}

Despite the great advances in control theory, most industrial applications are still lagging behind the current linear and nonlinear control methods by utilising simple PID control. Very few commercially available controllers - including industrial automation - are more sophisticated than the conventional linear controller. Proportional-Integral-Derivative (PID) control has proven successful for linear plants, but has limited effectiveness in nonlinear systems. Nonlinear control methods have performed very successfully in simulations and in experimental settings, yet the spread of these methods in industry is very slow due to their complexity. The highest level of controller complexity that a human operator is able to tune and troubleshoot is represented by a conventional (linear) controller. Often, the plant dynamics can only be described by a complex mathematical model. Physical imperfections, noise, sensor run-out and plant nonlinearities also contribute to the modelling inaccuracies for which robust, nonlinear control methods would be required. A good match for these requirements is fuzzy logic, which is based on human expressions rather than mathematical model of the plant. Fuzzy logic has proven to be very successful in settings where the plant parameters are unknown or ill-defined, time-varying or lacking accurate mathematical model. Fuzzy inputs have been used only in a few research works, yet this area of fuzzy logic control (FLC) is quickly emerging. This work aims to fill a gap in the application of fuzzy inputs for attenuation of persistent disturbances.

\section{FUZZY LOGIC CONTROLLER}

Fuzzy logic originates from the idea that most physical situations cannot be described using classical, two-valued (true-false) logic alone. There are many advantages of FLCs such as the ability to translate imprecise expressions to mathematical form, easier implementation, smoother and more robust controller operation compared to classical controllers and the ability to control unstable plants. Mamdani ${ }^{1}$ reported the first practical demonstration of a FLC to control a steam plant, which was soon followed by a variety of applications ranging from engineering to life sciences. In the following paragraph a brief review of the research on fuzzy logic in magnetic bearings is being presented as well as the works dealing with fuzzy inputs.

Hung $^{2,3}$ described the development of an FLC to adjust the gain of the PID controller's output for better compensation of the magnetic bearings' nonlinear effects. The simulations have shown that the linear PID controller benefits from the fuzzy controller in gaining more stability and robustness, providing stable operation and in the whole clearance. In Chen's work ${ }^{4}$ the derivative gain of a conventional PID controller was modified using an FLC. The FLC was designed in such a way that it modified the gain of the PID controller thus providing higher damping at high frequencies. Using FLC, the modified controller has also shown improved frequency-response. A similar approach was described by Koskinen, ${ }^{5,6}$ where the performance of a linear PID controller was improved using a fuzzy derivative gain adjusting method. It was also shown that the modified fuzzy PID (FPID) controller is less sensitive to vibration noise. In Hartavi's work ${ }^{7}$ the fuzzy logic controller has shown better response than the conventional PID, although the effects of tuning the fuzzy controller have not been addressed. Habib's paper ${ }^{8}$ discusses the effects of adjusting the fuzzy controller's parameters in some detail, although the fuzzy algorithm is only used to adjust a PID controller's gains. Most of the theoretical as well as experimental work on fuzzy logic control assumes crisp numerical inputs. This assumption is great simplification of the original fuzzy logic theory, which allows fuzzy set inputs. In general, a singleton, a crisp number is a one-dimensional, 